\title{
SOME OBSERVATIONS ON CHRONIC CLOSED-ANGLE GLAUCOMA*
}

\author{
BY \\ WALLACE S. FOULDS AND CALBERT I. PHILLIPS \\ From the Glaucoma Clinic, Institute of Ophthalmology, University of London \\ Director of Research: Sir Stewart Duke-Elder
}

AT WHAT stage in its course closed-angle glaucoma may be said to have become chronic is difficult to define. When, however, pathological cupping of the optic disc and visual field loss have occurred in an eye with closedangle glaucoma, the disease may reasonably be said to have reached the chronic stage. The aqueous outflow in such an eye is usually regarded as being permanently impaired by the development of goniosynechiae. It is the purpose of this communication to show that, in some cases, this advanced stage of chronic closed-angle glaucoma may result from closure of the filtration angle by irido-corneal contact alone, without the development of either synechiae or permanent trabecular change.

Barkan (1938) has stated that contact between the root of the iris and the entrance to the angle will, if transient, result in a rise of pressure of short duration; if the contact be not soon relieved by medication or operation, permanent organic adhesions will result. Grant (1951) has shown that periods of transient closure of the angle are associated with periods of reduced outflow of aqueous.

It has already been suggested (Phillips, 1956) that, in cases of "subacute" closed-angle glaucoma, the uppermost part of the angle of the anterior chamber is closed by irido-corneal contact before any attacks of hypertension occur, and that the area so obstructed advances downwards nasally and temporally until a critical amount of angle remains open below, the lowermost limits of this obstruction varying from time to time depending on such factors as pupillary dilatation.

While closure of the angle in cases of subacute closed-angle glaucoma was being investigated by means of the darkroom-outflow test (Foulds, 1956), it was noted that in certain cases the facility of aqueous outflow, measured by tonography (Grant, 1951), was lower than normal at the start of the test, although the ocular tension was within the accepted limits of normality. Moreover, the facility of aqueous outflow could be further reduced by a period in the dark, or could be increased by the instillation of a miotic, indicating an obstruction to aqueous outflow of variable degree. The following example illustrates this.

Case 1, a male aged 63, had developed an acute hypertensive episode in the right eye early in 1954. The attack responded to miotic therapy and thereafter the patient had been instilling pilocarpine 2 per cent. twice daily into this eye. In June 1955, the vision in the 
right eye was 6/9 unaided and the visual field full. Gonioscopy revealed a "very narrow angle, closed above but just open below". The ocular tension was found to be $28 \mathrm{~mm}$. $\mathbf{H g}$ some 7 hours after the instillation of pilocarpine 2 per cent.; the facility of aqueous outflow was $\mathbf{0} \cdot 10$. One hour in the dark caused the ocular tension to rise to $34 \mathrm{~mm} . \mathrm{Hg}$ while the facility of aqueous outflow fell to 0.05 . Immediately after the instillation of pilocarpine 2 per cent. the ocular tension was found to be $28 \mathrm{~mm}$. $\mathrm{Hg}$ but the facility of outflow had risen to 0.14. A further hour in the dark resulted in a rise of ocular tension to $30 \mathrm{~mm}$. $\mathrm{Hg}$ and a fall in the facility of outflow to 0.08 .

The results in this case suggested that, even on treatment with miotics, part of the angle of the anterior chamber was constantly obstructed by irido-corneal contact, the limits of which were variable; for most of the time the outflow of aqueous was as low as that found in cases of well-established chronic simple glaucoma. However, the fact that the facility of aqueous outflow immediately after the instillation of pilocarpine rose just to within normal limits suggested that some area of the angle was not permanently obstructed. The low outflow readings obtained at other times suggested that this type of case might eventually develop cupping of the optic disc and field loss without the appearance of permanent goniosynechiae.

Proof of this would be possible if the ocular tension in a well-developed case of chronic closed-angle glaucoma with advanced visual field loss and cupping of the optic disc could be relieved by a peripheral iridectomy and the angle of the anterior chamber subsequently shown to be open and free from goniosynechiae. A normal outflow factor post-operatively would indicate the absence of permanent trabecular change. Two cases of this type are described below:

Case 2, a male, aged 49, complained of attacks of blurred vision in the right eye for one year. He was found to have corneal oedema in the affected eye and an ocular tension of $60 \mathrm{~mm}$. Hg (Schiötz) which was reduced by miotics to $48 \mathrm{~mm}$. Hg. Gonioscopy revealed a very narrow angle apparently entirely closed. The optic disc was pathologically cupped and the visual field constricted to within $10^{\circ}$ of the fixation point (Fig. 1).

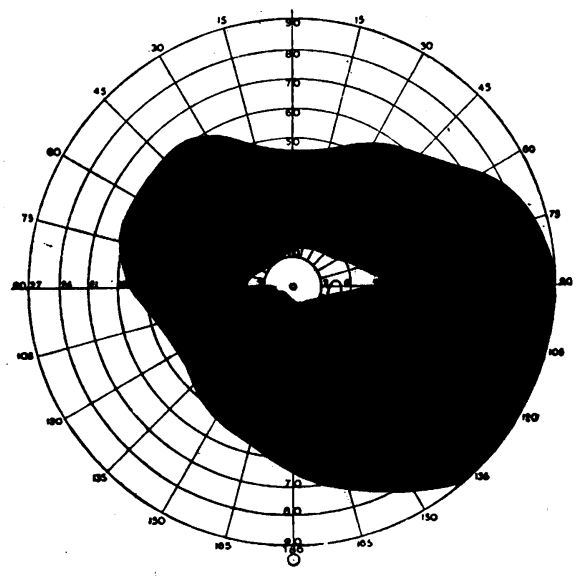

Fig. 1.-Field of vision of Case 2 before operation. 
A peripheral iridectomy was performed, after which the angle of the anterior chamber could be seen to be open and free from goniosynechiae (Fig. 2). The ocular tension thereafter did not rise above $20 \mathrm{~mm}$. $\mathrm{Hg}$ and the facility of aqueous outflow was $\mathbf{0 \cdot 2 5}$.

FIG. 2.-Post-operative gonioscopic appearance of the angle in Case 2. The angle is open in all sectors. The pre - operative appearance of the angle was similar to that in Case 3 (see Fig. 4).

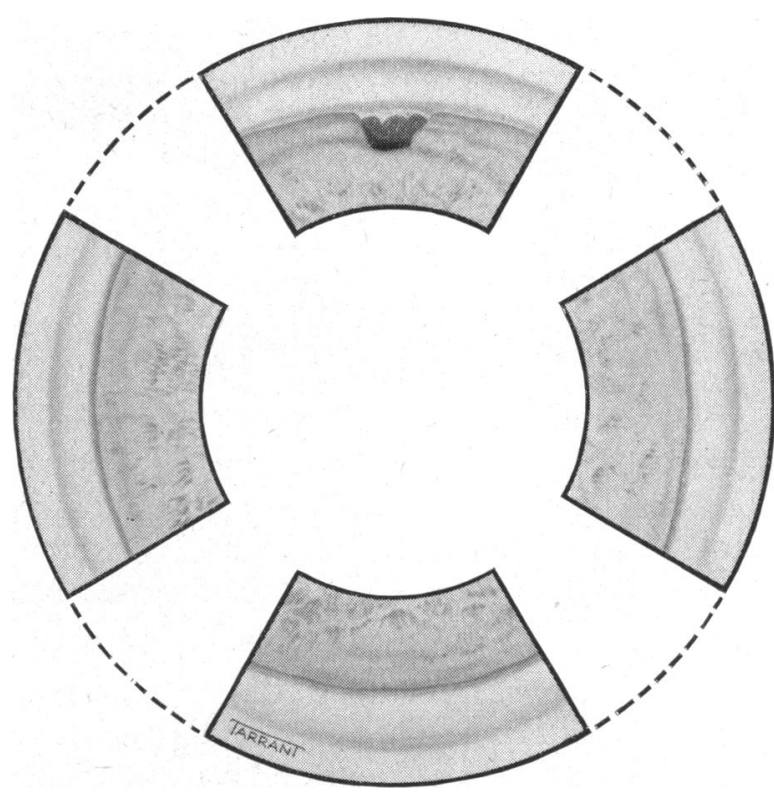

Case 3, a male aged 72, complained of discomfort in the right eye and failing vision for nine months. The ocular tension was found to be $70 \mathrm{~mm}$. $\mathrm{Hg}$, reduced by miotics to between 30 and $40 \mathrm{~mm}$. Hg. The optic disc was deeply cupped and the visual field showed nasal loss within $5^{\circ}$ of the fixation point, together with a typical Bjerrum scotoma (Fig. 3).

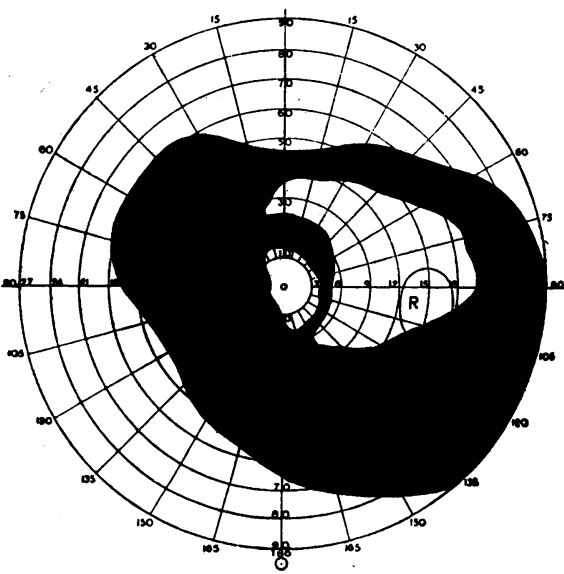

Fig. 3.-Field of vision of Case 3 before operation.

Gonioscopy shortly after the instillation of eserine 0.5 per cent. and pilocarpine 2 per cent. revealed a narrow angle, open in the lower segment but closed in the upper (Fig. 4, opposite). The facility of aqueous outflow at that time was 0.14 in the affected (compared with 0.20 in the unaffected) eye. 


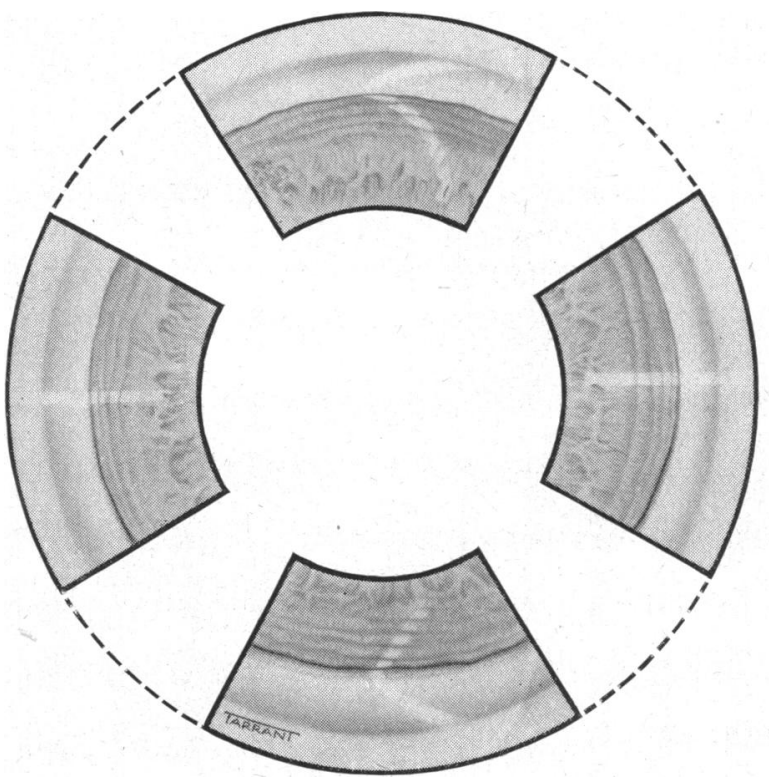

Fig. 4.-Gonioscopic appearance of the angle of the anterior chamber in Case 3 before operation. It is impossible to be certain whether the closure of the angle above is due to goniosynechiae or to irido-corneal contact.

A peripheral iridectomy was performed on the right eye, after which the previously closed sector of the angle could be seen to be open and free from goniosynechiae (Fig. 5). The ocular tension remained in the region of $20 \mathrm{~mm}$. $\mathrm{Hg}$ while facility of aqueous outflow was $\mathbf{0} \cdot 28$.

Fig. 5.-Gonioscopic appearance of the angle of the anterior chamber in Case 3 after a peripheral iridectomy had been performed. It is now obvious that the pre-operative closure of the angle was due to irido-corneal contact (see Fig. 4).

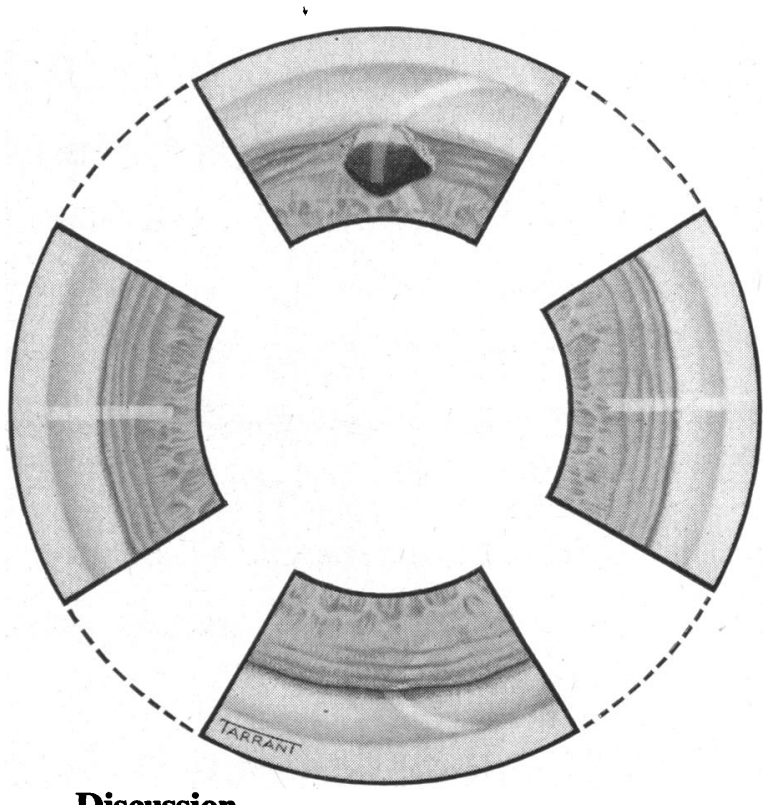

Discussion

The case histories described above show that chronic closed-angle glaucoma may be caused by irido-corneal contact extending so far round the angle 
of the anterior chamber that no amount of miosis will increase the outflow of aqueous to normal.

In the angle of an anterior chamber of normal depth goniosynechiae are easily seen. In a narrow angle the great difficulty in differentiating goniosynechiae from irido-corneal contact or merely extreme narrowness of the angle has already been pointed out (Phillips, 1956). Indeed, the differential diagnosis must depend much less on the objective appearances of the angle than on the results of provocative tests, outflow estimations, response of tension to miotics, and the history of the patient: the absence of any acute hypertensive episodes and the shortness of the history of symptoms are of great importance.

Since the criteria for diagnosis are so imprecise, failure of peripheral iridectomy to cure a case of chronic closed-angle glaucoma will sometimes occur even if the history seems to favour irido-corneal contact as the cause. One such case is recorded below. Possible failure was anticipated as closure of the angle had probably been present for 3 years and the iridectomy was done at 10 o'clock (right eye) so that if necessary a subsequent drainage operation might be performed at 12 o'clock.

Case 4, a female aged 51, first attended hospital when aged 44 with a complaint of "haloes" in the right eye. The left was amblyopic (a concomitant convergent strabismus being present). Miotic therapy was advised. At the age of 48 , the right eye, which had a visual acuity of $6 / 5$ with correction, showed a tension which was only once below $30 \mathrm{~mm}$. $\mathrm{Hg}$ in spite of miotics: all other tension readings were over $40 \mathrm{~mm}$. $\mathrm{Hg}$. Gonioscopy revealed a "very narrow" angle. The right field was full. Operation was advised for the right eye but was refused.

By the age of 51, the right eye showed definite supero-nasal field loss, a tension never less. than $32 \mathrm{~mm}$. $\mathrm{Hg}$ even after strong miotic drops, an angle blocked from 10 to 4 o'clock and a "border-line" outflow factor of $\mathbf{0} \cdot 12$. A peripheral iridectomy converted the "narrow" lower sector of the angle to "medium", no change appearing in the upper which must therefore have been closed by goniosynechiae. The tension remained raised and the outflow $\mathbf{0} \cdot 12$. Drainage operation was subsequently performed.

This publication has been prepared in order to prove that irido-corneal contact may be a cause of advanced chronic closed-angle glaucoma. So many difficulties arise in the differential diagnosis of goniosynechiae when the angle is narrow, that peripheral iridectomy in cases of chronic closedangle glaucoma apparently due to irido-corneal contact, should be used only with the greatest caution. Indeed, the possibility of its failure even in the most carefully chosen case of chronic closed-angle glaucoma serves to underline the importance of performing peripheral iridectomy in the early stages of closed-angle glaucoma before the chronic stage is reached. On the other hand, if a drainage operation is performed on all cases of chronic closedangle glaucoma, some eyes will be subjected to an unnecessarily major procedure. 


\section{Summary}

Evidence has been presented to show that advanced chronic closed-angle glaucoma with field loss and cupping of the optic disc may be caused not only by goniosynechiae, but also by irido-corneal contact.

Successful treatment of the latter is possible by peripheral iridectomy, whereas a drainage operation is required for the former. Gonioscopic discrimination between irido-corneal contact and goniosynechiae in a narrow angle is impossible, so that more reliance must be placed on the liability of tension and outflow in response to miotics, the absence of previous acute hypertensive episodes, and the shortness of the history of symptoms, in diagnosing the type due to irido-corneal contact. The existence of this diagnostic problem, which is sometimes insoluble, underlines the importance of discovering cases of closed-angle glaucoma long before chronicity becomes established, i.e. in the stage when peripheral iridectomy is almost certain to be curative.

We gratefully acknowledge the helpful criticism given by Sir Stewart Duke-Elder in the preparation of this paper.

The clinical details of patients have been made available for publication by kind permission of Mr. D. P. Greaves, Mr. S. J. H. Miller, and Mr. J. G. Milner. We wish to record thanks to Mr. T. Tarrant of the Department of Medical Illustration, Institute of Ophthalmology, for his preparation of the drawings.

\section{REFERENCES}

BARKAN, O. (1938). Amer. J. Ophthal., 21, 1102.

FouLDs, W. S. (1956). Trans. ophthal. Soc. U.K., 76, 83.

Grant, W. M. (1951). A.M.A. Arch. Ophthal., 46, 113.

PHILlIPS, C. I. (1956). British Journal of Ophthalmology, 40, 136. 